\title{
Hematoma duodenal posvideoendoscopia digestiva alta para diagnóstico de enfermedad de injerto contra huésped en pediatría: a propósito de dos casos

\author{
Duodenal hematoma after upper gastrointestinal endoscopy in pediatric graft vs \\ host disease: Report of two cases
}

\author{
Dra. Georgina Bergero ${ }^{a}$, Dra. Daniela Frangi ${ }^{b}$, Dra. Luciana Lerendeguic, Dra. Verónica Busoni ${ }^{b}$, \\ Dr. Pablo A. Lobos c, Dra. Marina Orsib y Dr. Julián Llera ${ }^{a}$
}

\begin{abstract}
RESUMEN
La enfermedad de injerto contra huésped es una complicación grave que se presenta después del trasplante de médula ósea, con morbilidad y mortalidad elevadas. El patrón de oro para evaluar su compromiso gastrointestinal es la endoscopia digestiva alta y baja con toma de biopsia. El desarrollo de hematoma duodenal intramural es una complicación poco frecuente asociada con este procedimiento.

Se presentan dos casos de hematoma duodenal intramural posendoscopia en pacientes con trasplante y sospecha de enfermedad injerto contra huésped que presentaron un cuadro agudo de dolor abdominal y sangrado intestinal. El diagnóstico se realizó por tomografía y recibieron tratamiento conservador, con un resultado favorable.

En ambos casos, el diagnóstico de enfermedad injerto contra huésped gastrointestinal sehizo a través de las biopsias colónicas con histología duodenal normal, lo que sugiere evitar la toma de muestras duodenales para prevenir esta grave complicación en pacientes dealto riesgoy, deestemodo, disminuir la morbilidad. Palabras clave: hematoma, enfermedades duodenales, endoscopia, enfermedad de injerto contra huésped.
\end{abstract}

\section{ABSTRACT}

Graft versus host disease is a serious complication that occurs following bone marrow transplant with significant morbidity and mortality. The gold standard to diagnose gastrointestinal graft versus host disease is upper and lower gastrointestinal endoscopy with histological validation. The development of intramural duodenal hematoma is a rare complication associated with this procedure.

a. Servicio de Clínica Pediátrica, Departamento de Pediatría.

b. Servicio de Gastroenterología y Hepatología Pediátrica.

c. Servicio de Cirugía y Urología Pediátrica, Departamento de Pediatría.

Hospital Italiano de Buenos Aires. Ciudad Autónoma de Buenos Aires, Argentina.

Correspondencia:

Dra. Georgina Bergero: bergerogeorgina@gmail.com

Financiamiento: Ninguno.

Conflicto de intereses: Ninguno que declarar.

Recibido: 2-11-2020

Aceptado: 7-4-2021
We present two cases of intramural duodenal haematoma after duodenal biopsies in bone marrow transplant patients that presented clinically with severe abdominal pain and intestinal bleeding. In both cases, CT scans confirmed the diagnosis and they were treated conservatively with favorable outcomes. Final diagnosis of gastrointestinal graft versus host disease was based on the colonic samples with normal duodenal histoarchitecture, which could lead to avoiding duodenal samples in future patients in order to prevent this serious complication and thus diminish morbidity.

Key words: hematoma, duodenal diseases, endoscopy, graft vs host disease.

http: / / dx.doi.org/10.5546/aap.2021.e513

Cómo citar: Bergero G, Frangi D, Lerendegui L, Busoni V, et al. Hematoma duodenal posvideoendoscopia digestiva alta para diagnóstico de enfermedad de injerto contra huésped en pediatría: a propósito de dos casos. Arch Argent Pediatr 2021;119(5):e513-e517.

\section{INTRODUCCIÓN}

La enfermedad de injerto contra huésped (EICH) es una complicación grave que puede presentarse luego del trasplante de médula ósea (TMO), con una morbilidad y mortalidad elevadas. ${ }^{1}$ Esta entidad suele aparecer dentro de los 100 primeros días posteriores al TMO y se considera que es la principal causa de muerte en más del $20 \%$ de los pacientes. Su génesis se explica por la activación de los linfocitos $\mathrm{T}$ del donante que reconocen las moléculas del sistema HLA o péptidos presentes en la superficie de las células presentadoras de antígenos del receptor. Este proceso tiene como órganos diana la piel, el hígado y el intestino. ${ }^{2}$ Cuando el tracto gastrointestinal está involucrado, a menudo se presenta con diarrea secretora, anorexia, vómitos $\mathrm{y}$, en casos graves, dolor abdominal y sangrado gastrointestinal. ${ }^{1}$

El patrón de oro para el diagnóstico de la EICH gastrointestinal (EICH-GI) es la videoendoscopia alta y baja con toma de biopsia. ${ }^{3}$ El desarrollo de hematoma duodenal intramural (HDI) es una complicación poco frecuente asociada a 
este procedimiento. Aunque se desconoce su incidencia, se estima que se produce en alrededor de 1:2000 endoscopias gastrointestinales altas,, 45 con una mayor proporción en pacientes pediátricos. ${ }^{6,7}$ Es excepcional que se presente en pacientes sin patología previa; se cree que la anatomía duodenal, el tipo de anestesia utilizada y la posición en la que se realiza la endoscopia podrían ser factores potencialmente asociados. ${ }^{8-10}$ Sin embargo, el hematoma duodenal se ha relacionado frecuentemente con diferentes comorbilidades tales como coagulopatía, trombocitopenia, enfermedades oncohematológicas y malnutrición. Desde este punto de vista, el TMO parece desempeñar un papel central como factor predisponente. ${ }^{8}$

Se describen dos casos de hematoma duodenal tras la realización de biopsia duodenal endoscópica para evaluar EICH-GI luego de TMO.

\section{Caso 1}

Paciente de 10 años con antecedente de leucemia mieloide aguda, que cursa el segundo TMO. En el día 18 postrasplante, presentó diarrea y fiebre sin evidencia de infección. Debido a la persistencia de los síntomas, se realizó videoendoscopia digestiva alta y baja con toma de muestras para biopsia para evaluar la presencia de EICH-GI. Durante las primeras horas posoperatorias, el paciente experimentó hematemesis y dolor abdominal agudo, sin signos de inestabilidad hemodinámica. El hematocrito se mantuvo estable y no se evidenciaron signos de perforación intestinal. Sin embargo, 12 horas después del procedimiento endoscópico, el dolor abdominal y los vómitos se intensificaron.

El marcado aumento de los valores de amilasa (1900 UI/L) y lipasa (4580 UI/L), junto con una disminución tanto de los niveles de hematocrito como de hemoglobina, motivó la realización de una tomografía computarizada con contraste intravenoso, que mostró un gran hematoma parietal submucoso ( $39 \mathrm{~mm} \times 43 \mathrm{~mm} \times 82 \mathrm{~mm}$ ) localizado entre la segunda y la tercera porción del duodeno, causando una obstrucción tanto del colédoco como del conducto pancreático (Figura 1).

Se colocó una sonda nasogástrica descompresiva. Los valores de lipasa y amilasa se normalizaron dos días después. Se confirmó la presencia de EICH- GI en las biopsias de colon, sin anomalías histológicas en las muestras duodenales, y se inició tratamiento acorde. Se administró nutrición parenteral total durante un período de 20 días, tras el cual se reinició el aporte por vía oral de forma progresiva hasta lograr alimentación enteral completa. Se utilizó la ecografía como método no invasivo para el seguimiento del tamaño del hematoma. El paciente evolucionó de manera favorable, con resolución completa del hematoma un mes posterior al evento.

\section{Caso 2}

Paciente de 20 meses con antecedente de leucemia mielomonocítica juvenil y TMO histoidéntico no relacionado, que recibió tratamiento profiláctico con tacrolimus como prevención de EICH postrasplante. El día 14 postrasplante presentó diarrea, a la que agregó hematoquecia en las siguientes semanas. Se decidió realizar una endoscopia digestiva alta y colonoscopia con toma de muestras para biopsia para descartar EICH-GI. Cinco horas después

FIgURA 1. Tomografía computarizada abdominal durante evento agudo posvideoendocopia digestiva alta (caso 1)

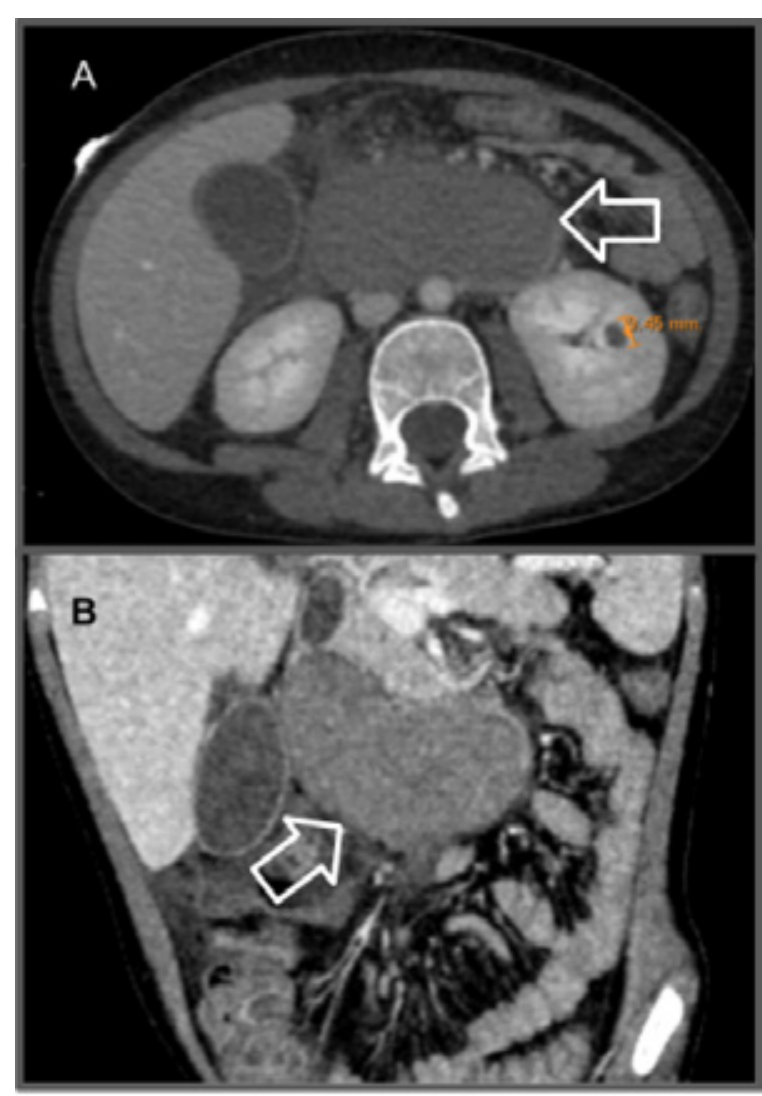

Se observa la presencia de un gran hematoma dentro de la pared del duodeno (flechablanca). A: vista axial; B: vista frontal. 
del procedimiento, el paciente presentó dolor y distensión abdominal asociado a inestabilidad hemodinámica y anemia. No se identificaron signos de perforación en la radiografía abdominal. Se diagnosticó la presencia de un hematoma duodenal ( $28 \mathrm{~mm} \times 65 \mathrm{~mm} \times 55 \mathrm{~mm}$ ) por medio de una tomografía abdominal, con realización posterior de angiografía que permitió excluir sangrado activo. El manejo conservador se decidió después de una compensación clínica inicial en la unidad de cuidados intensivos pediátricos. Presentó melena al reiniciar el tránsito intestinal.

Las biopsias duodenales resultaron ser normales, pero se confirmó EICH en las muestras de colon, y se indicó tratamiento acorde. Un mes más tarde, se realizó una nueva tomografía abdominal, que mostró una disminución moderada del hematoma (Figura 2). Se reinició la nutrición enteral después de 40 días de reposo digestivo y nutrición parenteral; se logró restaurar una alimentación enteral completa el día 50 después del evento. Durante el seguimiento, se evidenció persistencia del hematoma por ecografía a los dos meses del evento, pero sin repercusión clínica. Se realizó control periódico del paciente, quien no presentó intercurrencias. Una nueva ecografía, realizada a los 9 meses del evento, confirmó la resolución completa del hematoma.

En la Tabla 1 se muestran las principales características clínicas de ambos casos.

\section{DISCUSIÓN}

El desarrollo de un HDI después de una biopsia duodenal endoscópica es una complicación infrecuente. Se ha descrito su asociación con trastornos de la coagulación, leucemia y trasplante de órganos, pero, en los últimos años, se encontró que el HDI posprocedimiento es significativamente más frecuente en niños con TMO y con sospecha de EICH. ${ }^{10}$

El HDI suele causar síntomas gastrointestinales obstructivos, como vómitos biliosos y dolor abdominal intenso, que se presentan en las primeras horas o días después del procedimiento endoscópico. El hematoma también puede obstruir la papila duodenal, lo que lleva al desarrollo de pancreatitis y colestasis. Los dos pacientes aquí descritos se presentaron clínicamente con dolor abdominal graves, náuseas y sangrado digestivo. El primer paciente también presentó colestasis asociada y aumento de las enzimas pancreáticas. Estos hallazgos se han informado en la literatura en cerca del $50 \%$ de los pacientes. ${ }^{10}$ Los síntomas clínicos, asociados a estudios por imágenes como la ecografía, la tomografía computarizada abdominal o la resonancia magnética, confirman el diagnóstico. ${ }^{10-12}$ La tomografía abdominal fue la prueba diagnóstica de elección durante la fase aguda en los dos casos. En el segundo caso, debido a la presencia de líquido libre en la

FIGURA 2. Evolución imagenológica de hematoma duodenal (caso 2)
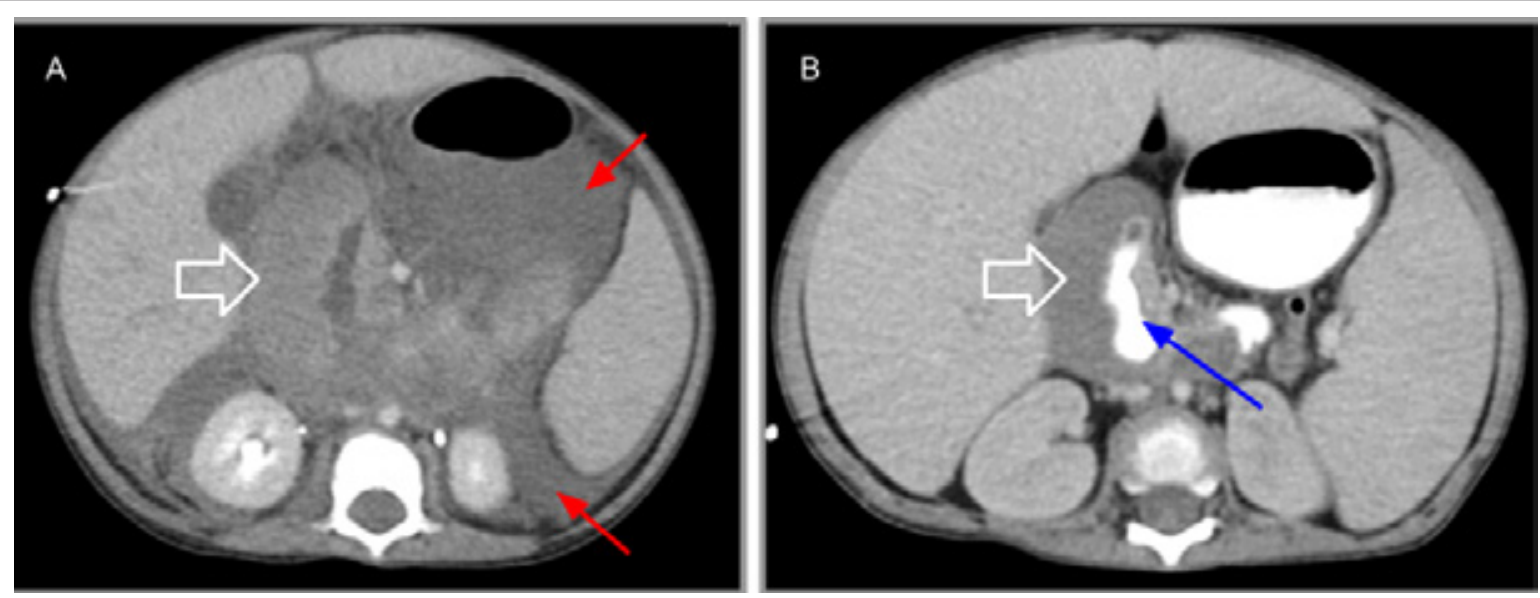

A: tomografía computada durante el cuadro agudo posvideoendoscopia digestiva alta. Se observa líquido libre (flechas rojas) y hematoma duodenal con oclusión de la luz duodenal (flecha blanca). B: tomografía computarizada diferida. Se observa pasaje de contraste (flecha azul) a través de la luz duodenal con reducción del hematoma mural duodenal (flecha blanca). 
cavidad abdominal y a la anemia aguda grave temprana, también se realizó una angiografía para descartar una hemorragia activa. Se realizó un seguimiento con ecografías para evitar una alta exposición a la radiación. La resonancia magnética podría ser otro método alternativo de diagnóstico por imágenes.

No existe consenso en cuanto al tratamiento del HDI, aunque, con base en los datos publicados, se debería preferir un tratamiento conservador. La terapia de reemplazo hidroelectrolítica, la descompresión abdominal con sonda nasogástrica, el manejo del dolor y el apoyo con nutrición parenteral son suficientes como tratamiento de apoyo hasta la resolución del hematoma. La bibliografía notifica una resolución completa de los síntomas, en general, en un plazo de 2 a 3 semanas, sin necesidad de intervención quirúrgica. ${ }^{12,13}$ En nuestra experiencia, el manejo conservador fue posible en ambos casos, a pesar de la presentación inusualmente grave del segundo caso.

Varios estudios en adultos sugieren que la endoscopia alta no aumenta de manera significativa la precisión diagnóstica de la EICH digestiva en comparación con la ileocolonoscopia. ${ }^{10}$ También se ha notificado en la literatura que la combinación de toma de muestras de biopsia del área rectosigmoidea con el tubo digestivo superior tendría una sensibilidad

TABLA 1. Análisis comparativo de las principales características clínicas de ambos casos

\begin{tabular}{|c|c|c|}
\hline Características clínicas principales & Caso 1 & Caso 2 \\
\hline Sexo y edad & Masculino, 10 años & Masculino, 20 meses \\
\hline Enfermedad de base & Leucemia mieloide aguda & Leucemia mielomonocítica juvenil \\
\hline Tipo de TMO & $2^{\circ} \mathrm{TMO}$, haploidéntico & $1^{\circ} \mathrm{TMO}$, histoidéntico no relacionado \\
\hline Profilaxis para EICH & No & Sí, con tacrolimus \\
\hline $\begin{array}{l}\text { Síntomas clínicos que orientan a } \\
\text { la EICH intestinal }\end{array}$ & Diarrea & Diarrea con sangre \\
\hline $\begin{array}{l}\text { Recuento de plaquetas previo } \\
\text { al procedimiento endoscópico }\end{array}$ & $\underset{\mathrm{a}}{41200 / \mathrm{mm}^{3 \mathrm{a}}}$ & $22500 / \mathrm{mm}^{3}$ \\
\hline $\begin{array}{l}\text { Macroscopia de la mucosa duodenal } \\
\text { durante la endoscopia }\end{array}$ & Sin anormalidades & ducción del número de pliegues duodenales \\
\hline $\begin{array}{l}\text { Macroscopia de la mucosa colónica } \\
\text { durante la endoscopia }\end{array}$ & Sin anormalidades & $\begin{array}{c}\text { Pérdida completa del patrón vascular y } \\
\text { petequias hemorrágicas submucosas } \\
\text { Hiperplasia nodular }\end{array}$ \\
\hline Toma de biopsia duodenal & Sí & Sí \\
\hline Diagnóstico de EICH en biopsia duodenal & No & No \\
\hline Diagnóstico de EICH en biopsia colónica & Sí & Sí \\
\hline \multicolumn{3}{|l|}{ Características de la complicación } \\
\hline $\begin{array}{l}\text { Cuadro clínico instaurado dentro de } \\
\text { las primeras horas posendoscopia }\end{array}$ & Sí & Sí \\
\hline Inestabilidad clínica & No & Sí \\
\hline Dolor abdominal & Sí & Sí \\
\hline Sangrado gastrointestinal & $\begin{array}{c}\text { Sí } \\
\text { (hematemesis) }\end{array}$ & $\begin{array}{c}\text { Sí } \\
\text { (melena) }\end{array}$ \\
\hline Análisis de laboratorio & $\begin{array}{c}\text { Caída tardía del hematocrito } \\
\text { (16 h después del evento) } \\
\text { Amilasa y lipasa aumentadas } \\
\text { (1900 UI/L y } 4580 \text { UI/L, respectivamente) }\end{array}$ & Anemia grave aguda temprana \\
\hline Tomografía computada & $\begin{array}{l}\text { Hematoma duodenal } \\
\text { (39 } \mathrm{mm} \times 43 \mathrm{~mm} \times 82 \mathrm{~mm})\end{array}$ & $\begin{array}{c}\text { Hematoma duodenal } \\
(28 \mathrm{~mm} \times 65 \mathrm{~mm} \times 55 \mathrm{~mm})\end{array}$ \\
\hline Tratamiento & SNG para descompresión y NPT & SNG para descompresión y NPT \\
\hline Tiempo hasta la alimentación completa & 20 días & 50 días \\
\hline
\end{tabular}

EICH: enfermedad de injerto contra huésped, TMO: trasplante de médula ósea, TC: tomografía computada, NPT: nutrición parenteral, SNG: sonda nasogástrica. 
similar para el diagnóstico de EICH-GI a la de la ileocolonoscopia sola. ${ }^{14}$ En esta misma línea, se habían comunicado resultados similares en un estudio prospectivo que incluía una población mixta de niños y pacientes adultos, lo que sugería, incluso, que si la evaluación endoscópica del rectosigma es altamente sugestiva de EICH podría no requerirse una evaluación más alta en pacientes con diarrea, sin síntomas digestivos superiores. ${ }^{15}$ En nuestros casos, la EICH-GI se confirmó en muestras de colon y, aunque en ambos pacientes se tomaron muestras para biopsia del duodeno, estas no mostraron signos de la enfermedad.

Aunque el HDI es una complicación poco frecuente después de una biopsia duodenal, es importante contar con un alto índice de sospecha, ya que un diagnóstico temprano conduce a un tratamiento adecuado y a un mejor resultado. En los pacientes con factores de riesgo y síntomas compatibles, se debe realizar una resonancia magnética o una tomografía computarizada para confirmar el diagnóstico. Dado que en ambos casos presentados el diagnóstico de EICH-GI fue confirmado en el colon, y en ninguno de ellos la biopsia duodenal mostró signos de la enfermedad, el alto riesgo de esta complicación sugiere evitar las biopsias duodenales en este tipo de pacientes, en particular cuando el aspecto endoscópico es normal, con el objetivo de disminuir la morbilidad asociada.

\section{REFERENCIAS}

1. Goker H, Haznedaroglu IC, Chao NJ. Acute graft-vs-host disease: pathobiology and management. Exp Hematol. 2001; 29(3):259-77.

2. Sullivan KM. Graft-vs.-Host-Disease. In: Blume KG, Forman SJ, Appelbaum FR (eds). Thomas' Hematopoietic cell transplantation. 3rd ed. Victoria, Australia: Blackwell Publishing; 2004.Págs.635-64.

3. Thomson M, Tringali A, Dumonceau J-M, Tavares M, et al. Paediatric Gastrointestinal Endoscopy: European Society for Paediatric Gastroenterology Hepatology and Nutrition and European Society of Gastrointestinal Endoscopy Guidelines. J Pediatr Gastroenterol Nutr. 2017; 64(1):133-53.

4. Guzman C, Bousvaros A, Buonomo C, Nurko S. Intraduodenal hematoma complicating intestinal biopsy: case reports and review of the literature. Am J Gastroenterol. 1998; 93(12):2547-50.

5. Sahn B, Anupindi SA, Dadhania NJ, Kelsen JR, et al. Duodenal Hematoma Following EGD: comparison with blunt abdominal trauma-induced duodenal hematoma. J Pediatr Gastroenterol Nutr. 2015; 60(1);69-74.

6. Diniz-Santos DR, de Andrade Cairo RC, Braga H, AraújoNeto C, et al. Duodenal hematoma following endoscopic duodenal biopsy: a case report and review of the existing literature. Can J Gastroenterol. 2006; 20(1):39-42.

7. Grasshof C, Wolf A, Neuwirth F, Posovszky C. Intramural Duodenal Haematoma after Endoscopic Biopsy: Case Report and Review of the Literature. Case Rep Gastroenterol. 2012; 6(1):5-14.

8. Hameed S, McHugh K, Shah N, Arthurs OJ. Duodenal haematoma following endoscopy as a marker of coagulopathy. Pediatr Radiol. 2014; 44(4):392-7.

9. Ramakrishna J, Treem WR. Duodenal hematoma as a complication of endoscopic biopsy in pediatric bone marrow transplant recipients. J Pediatr Gastroenterol Nutr. 1997; 25(4):426-9.

10. Sierra A, Ecochard-Dugelay E, Bellaïche M, Tilea B, et al. Biopsy-Induced Duodenal Hematoma Is Not an Infrequent Complication Favored by Bone Marrow Transplantation. J Pediatr Gastroenterol Nutr. 2016; 63(6):627-32.

11. Dunkin D, Benkov KJ, Rosenberg HK. Duodenal and rectal hematomas complicating endoscopic biopsy: use of sonography in pediatrics. J Ultrasound Med. 2009; 28(11):1575-80.

12. AntoniouD, ZarifiM, GentimiF, Christopoulos-Geroulanos G. Sonographic diagnosis and monitoring of an intramural duodenal hematoma following upper endoscopic biopsy in a child. J Clin Ultrasound. 2009; 37(9):534-8.

13. Clendenon JN, Meyers RL, Nance ML, Scaife ER. Management of duodenal injuries in children. J Pediatr Surg. 2004; 39(6):964-8.

14. Mårtensson T, Szakos A, Mellgren K, Toporski J, et al. Choice of Endoscopic Procedure in Children With Clinically Suspected Gastrointestinal Graft-versus-host Disease. J Pediatr Gastroenterol Nutr. 2018; 66(5):744-50.

15. Thompson B, Salzman D, Steinhauer J, Lazenby AJ, Wilcox CM. Prospective endoscopic evaluation for gastrointestinal graft-versus-host disease: determination of the best diagnostic approach. Bone Marrow Transplant. 2006; 38(5):371-6. 